\title{
Alpha-1-Antitrypsin-Mangel: Diagnose und Therapie der pulmonalen Erkrankung
}

\author{
Alpha-1-Antitrypsin Deficiency: Diagnosis and Therapy \\ of the Lung Disease
}

S. Schroth ${ }^{1}$, R. Koczulla ${ }^{1}$, C. Herr ${ }^{1}$, T. Greulich ${ }^{1}$, E. M. Walthers ${ }^{2}$, C. Vogelmeier ${ }^{1}$, R. Bals ${ }^{1}$

${ }^{1}$ Klinik für Innere Medizin mit Schwerpunkt Hämatologie, Onkologie und Immunologie,

Universitätsklinikum Gießen und Marburg, Philipps Universität Marburg, Marburg

${ }^{2}$ Klinik für Strahlendiagnostik, Universitätsklinikum Gießen und Marburg, Philipps Universität Marburg, Marburg

\section{Lernziele}

$\nabla$

Die Lernziele umfassen den Überblick über die pulmonalen Auswirkungen des Alpha-1-Antitrypsinmangels einschließlich Biologie, Epidemiologie, Symptomatik, Diagnostik und Therapie. Ein Schwerpunkt liegt auf dem diagnostischen Vorgehen und den Therapiemöglichkeiten. Eine zweiseitige Zusammenfassung enthält die wichtigsten Informationen zur Diagnose und Therapie.

\section{Biologische Grundlagen}

Das Alpha-1-Antitrypsin (AAT) wird kontinuierlich in der Leber produziert und in das Blut abgegeben. Es zählt zu den Akut-Phase-Proteinen und schützt als Proteinaseinhibitor körpereigene Proteine vor dem Verdau. In der Lunge werden aufgrund der großen Kontaktfläche mit der Atemluft ständig verschiedene Schutzmechanismen aktiviert, wozu auch die Freisetzung und Aktivierung von Proteasen gehört. Proteasen sind Enzyme, die andere Proteine spalten und so deren Funktion inaktivieren, aktivieren oder modifizieren. Neutrophile Granulozyten setzen beispielsweise die Protease Elastase frei, die in der Lunge an Abwehrprozessen beteiligt ist [1]. Neben vielen anderen Antiproteinasen stellt das AAT aufgrund der hohen Konzentration im Serum und im Gewebe eine wichtige Komponente im Proteasen/ Antiproteasen-Gleichgewicht dar.

Die Synthese des AAT erfolgt vor allem in Leberzellen, von wo aus das Protein in das Blut abgegeben wird $(\bullet$ Abb. 1) [2].

Über das Blut erfolgt eine Verteilung in die Gewebe des Körpers. Weiterhin wird AAT in Neutrophilen und Epithelzellen der Lunge gebildet. Die Größe des AAT-Proteins beträgt $52 \mathrm{kDa}$. Das Gen, das für AAT kodiert, wird als SERPINA1 bezeichnet, ist 12,2 kb lang und besteht aus 7 Exons und sechs Introns [3-5]. Das Protein besteht aus 394 Aminosäuren, das aktive Zentrum des Enzyms befindet sich an der Aminosäure Methionin an der Po- sition 358 und wird innerhalb des Exons 5 codiert. Es existieren über 100 genetische Varianten, viele davon mit Krankheitswert.

Die Serumkonzentration liegt normalerweise bei $150-300 \mathrm{mg} / \mathrm{dl}$ (bzw. 20-52 $\mu \mathrm{mol} / \mathrm{l}$ ) und ist beim Vorliegen von Mutationen reduziert [7]. Zur Klassifizierung wird oft eine Kurzformel angewendet, bei der Pi (Proteinaseinhibitor) von den beiden Allelbezeichnungen gefolgt ist. Dabei sind M1-M6 die Normvarianten [6]. Bei Nullmutationen Pi Null wird kein Protein synthetisiert. Die meisten klinischen relevanten (95\%) Mangelzustände werden durch das homozygote Vorkommen der Z-Mutation verursacht, Pi ZZ (oder Pi Z). Weitere Mangelvarianten sind seltener anzutreffen: Pi SZ, Pi SS und andere. Der Serumspiegel bei Patienten mit beispielsweise dem Genotyp Pi ZZ ist mit $20-50 \mathrm{mg} / \mathrm{dl}$, bei Patienten mit dem Genotyp Pi SZ mit durchschnittlich 80 mg/dl stark

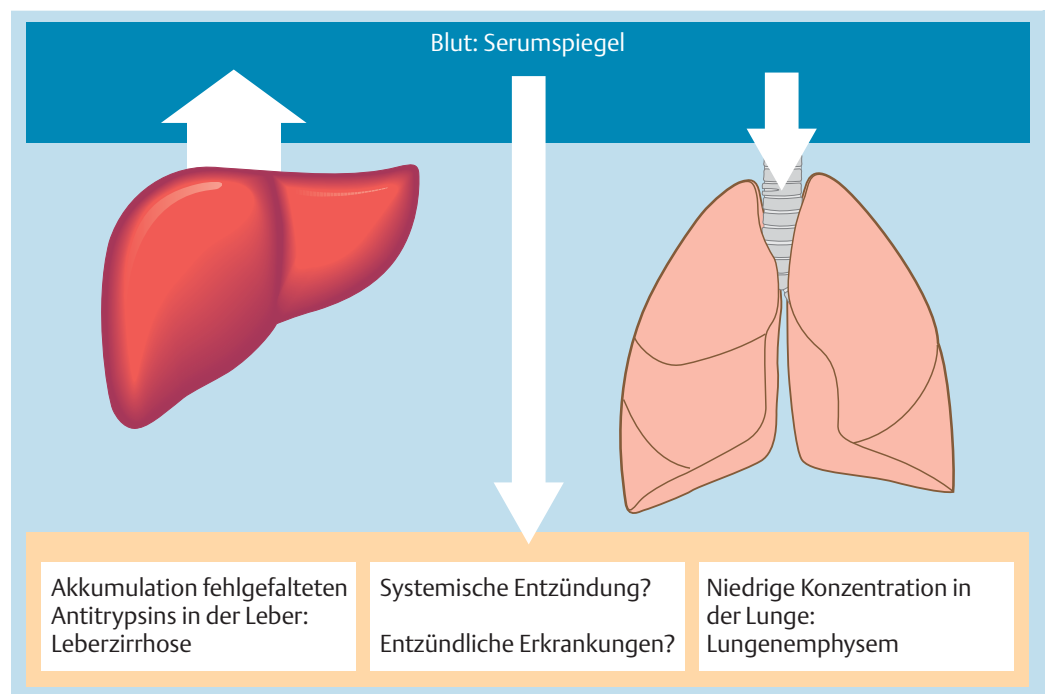

Abb. 1 Pathophysiologie der Leber- und Lungenerkrankung beim AATM. AAT wird in Hepatozyten produziert und in das Blut abgegeben, über das die Antiprotease die Lunge erreicht. Einige Mutationen führen über eine Fehlfaltung zu einer Akkumulation des AAT in Hepatozyten. Dies kann über die Induktion einer zellulären Stressreaktion zu einer Leberzirrhose führen. In der Lunge führt der Mangel an AAT zu einer ungehinderten Aktivität von neutrophiler Elastase. Dies resultiert in einer Schädigung des Lungengewebes. 
Tab. 1 Häufige Genotypen und der korrelierende Serumspiegel mit dem relativen Risiko, an einer COPD zu erkranken [7].

\begin{tabular}{|lllll|} 
Genotyp & AAT Serum Spiegel & $\begin{array}{l}\text { Risiko für eine } \\
\text { Lungenbeteiligung }\end{array}$ & $\begin{array}{l}\text { Risiko für eine } \\
\text { Leberbeteiligung }\end{array}$ \\
\hline Pi MM & $20-48$ & $150-350$ & kein Risiko & kein Risiko \\
\hline Pi MZ & $17-33$ & $90-210$ & minimales Risiko & minimales Risiko \\
\hline Pi SS & $15-33$ & $100-200$ & kein Risiko & kein Risiko \\
\hline PiSZ & $8-16$ & $75-120$ & geringes Risiko & minimales Risiko \\
\hline Pi ZZ & $2,5-7$ & $20-45$ & hohes Risiko & hohes Risiko \\
\hline Null & 0 & 0 & hohes Risiko & Abh. von der Mutation \\
\hline
\end{tabular}

erniedrigt. Tab. 1 gibt einen Überblick über die wichtigsten Genotypen und die daraus resultierenden Serumspiegel und klinischen Konsequenzen.

\section{Pathophysiologie \\ $\nabla$}

Der AAT-Mangel (AATM) ist eine genetisch vererbbare Prädisposition für verschiedene Erkrankungen. Einige Mutationen (beispielsweise $\mathrm{Z}$,

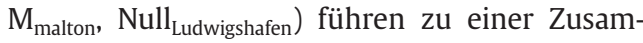
menlagerung fehlerhaft gefalteter AAT-Moleküle im Hepatozyten, was über die Entwicklung einer zellulären Stressreaktion zur Entwicklung einer Leberzirrhose führen kann ( $\bullet$ Abb. 1) [2]. Diese Synthesestörung führt auch zu einer verminderten Abgabe, weiterhin besitzen manche mutierte AAT-Proteine auch eine geringere Antiproteaseaktivität. Der Serumspiegel an AAT und nachfolgend die Konzentration in der Lunge ist eng mit dem vorliegenden Genotyp assoziiert ( Tab. 1). Je niedriger der Serumspiegel, desto höher ist die Wahrscheinlichkeit für das Auftreten einer COPD [8].

Bei vielen Mutationen kommt es durch die Synthesestörung zu einem niedrigen Serumspiegel und so zu einem Mangel an AAT in den peripheren Organen. In der Lunge kommt es durch ein gestörtes Gleichgewicht zwischen der Proteinase und AAT zu einem Überschuss an Proteaseaktivität. Hierdurch kommt es zu einer langsam fortschreitenden Zerstörung der Lungenstruktur. Auf diese Weise bildet sich ein basal betontes Lungenemphysem aus. Neben der Funktion als Antiprotease gibt es in letzter Zeit Hinweise auf die Funktion von AAT als entzündungshemmendes Protein [8]. Außerdem wurde beschrieben, dass die Z-Form des AAT auch chemotaktisch auf Neutrophile wirken kann und so auch zur Aufrechterhaltung der Entzündung in der Lunge beitragen könnte [9]. Neben der Lungen- und der Lebererkrankung gibt es weitere mit einem AATM assoziierte Krankheitsentitäten, wie die Pannikulitis und die c-ANCA Vaskulitis (z.B. Wegener'sche Granulomatose) [7].
Der Serumspiegel an AAT und nachfolgend die Konzentration in der Lunge ist eng mit dem vorliegenden Genotyp assoziiert. Je niedriger der AATSerumspiegel, desto höher ist die Wahrscheinlichkeit für eine Lungenschädigung.

\section{Epidemiologie \\ $\nabla$}

In Deutschland gibt es basierend auf den epidemiologischen Daten und Hochrechnungen ca. 8000 bis 12000 Individuen mit einem schweren AATM. Wie viele davon erkrankt sind, ist nicht bekannt. Aktuell sind etwa 2500 Patienten mit einem AATM bekannt. Die Häufigkeit wird in den mitteleuropäischen Ländern auf 0,01 und 0,02\% geschätzt $[10,11]$. In Nord- und Mitteleuropa stellt der Alpha-1-Antitrypsinmangel die häufigste autosomal vererbbare Krankheit dar, die zum Tode führen kann. Weltweit existieren nach Hochrechnungen 116 Millionen Genträger und 1,1 Millionen Personen mit einem schweren AATM [10]. Bei Risikokollektiven ist das Vorkommen eines AATM häufiger.

In Deutschland gibt es basierend auf den epidemiologischen Daten und Hochrechnungen ca. 8000 bis 12000 Personen mit einem schweren AATM. Für Pi MZ (Träger) ist die Häufigkeit ungefähr $1: 50$, für $\mathrm{Pi} Z Z$ ungefähr $1: 5000$ bis $1: 10000$.

\section{Symptomatik der Lungenmanifestation}

$\nabla$

Die Beschwerden einer AATM-bedingten COPD unterscheiden sich nicht von denen einer konventionellen COPD, allerdings treten die Beschwerden typischerweise früher (ab dem 35. Lebensjahr) auf und die Erkrankung zeigt einen schwereren Verlauf [4]. Die typischen ersten Beschwerden sind Husten und Belastungsdyspnoe $[12,13]$. Wegen der geringen Spezifität der Beschwerden und wohl wegen der Seltenheit der Erkrankung wird die Diagnose meist erst nach mehreren Jahren gestellt - durchschnittlich nach 4-6 Jahren [14]. In 20\% der Fälle findet sich ein hyperreagibles Bronchialsystem. Durch die mögliche Ausbildung von Bronchiektasien und dadurch entstehenden Retentionen werden Entzündungsreaktionen unterhalten. Auch bei der AATM-bedingten COPD komplizieren Exazerbationen den Krankheitsverlauf und bedingen eine klinisch relevante Verschlechterung. Im Verlauf der Erkrankung kann es zu einem respiratorischen Versagen im Sinn einer Hypoxie und dann im Weiteren auch zu einer Hyperkapnie bei Atempumpenversagen kommen.

Eine konkrete Vorhersage, wie sich bei bestehendem schwerem AATM (zum Beispiel Pi ZZ) Organmanifestationen entwickeln werden, ist nicht 
Indikationen für eine quantitative Bestimmung des AATSpiegels im Serum:

- Frühes Emphysem

- Emphysem ohne Risikofaktoren

- Basales Emphysem

- Unklare Lebererkrankung

- Nekrotisierende Panniculitis

- Anti-Proteinase 3 positive Vaskulitis

- Familienanamnese: Emphysem, AATD, Bronchiektasie, Leberkrankheiten, Panniculitis

- Bronchiektasie

Quantitative Bestimmung des AAT-Spiegels im Serum

AAT normal

AAT niedrig
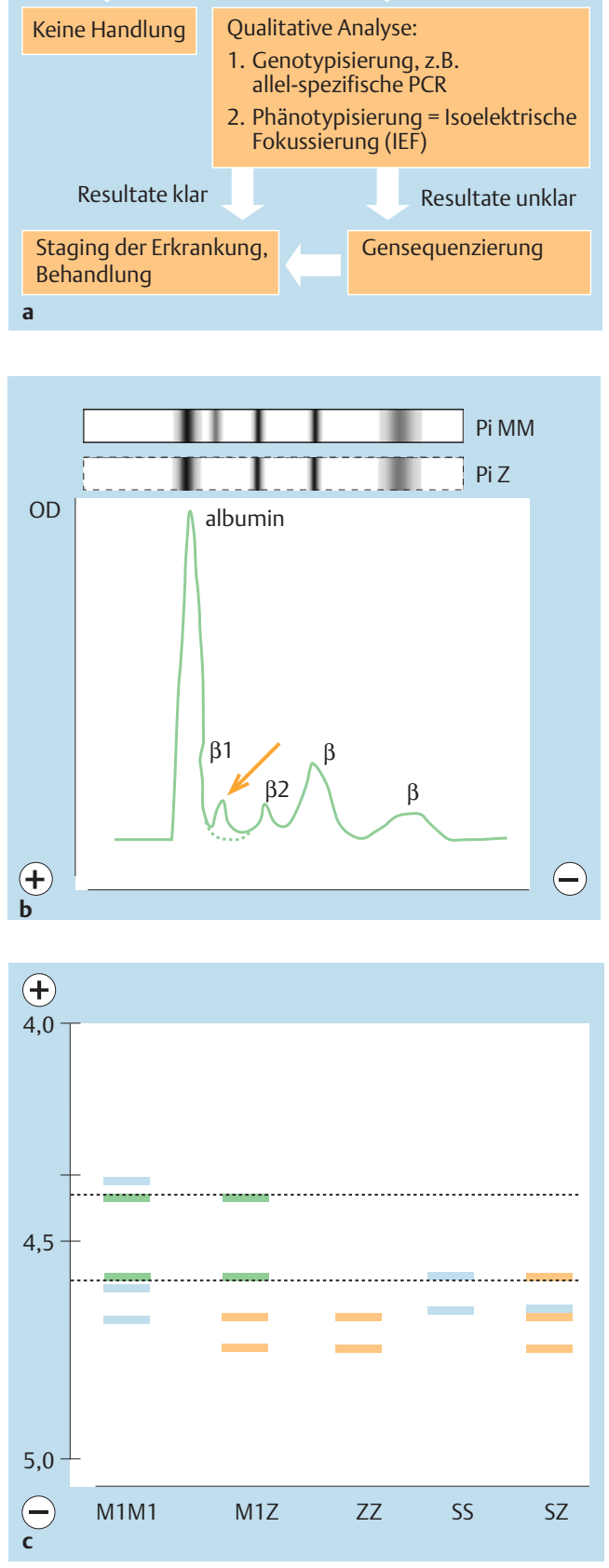

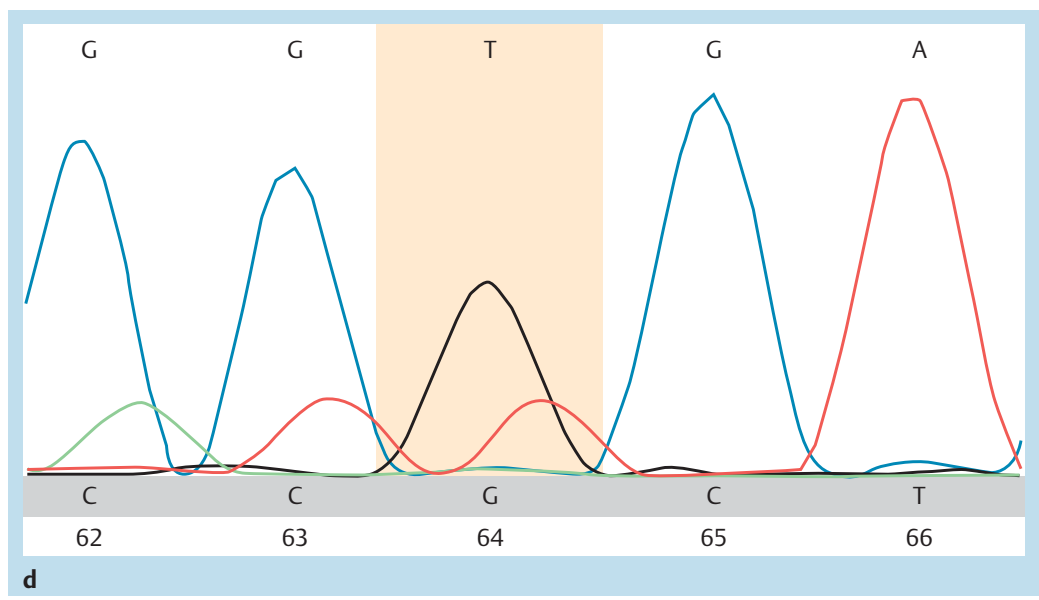

Abb. 2 Labordiagnose des AATM. a Ablauf der Diagnose des AATM. b Die Serumproteinelektrophorese ist ein klassisches Verfahren und kann durch Fehlen der alpha-1-Zacke auf einen AATM hinweisen. c Die isoelektrische Fokussierung dient zur Identifikation des Phänotyps. Proteine werden hierbei nach ihrem isoelektrischen Punkt aufgetrennt. Verschiedene Formen des AATProteins geben spezifische Muster. $\mathbf{d}$ Mittels der Sequenzierung kann in Einzelfällen die Sequenz des Gens analysiert werden, das für AAT kodiert. So können seltene oder neue Mutationen identifiziert werden. Das aktuelle Beispiel zeigt die Primärdaten des Sequenziergerätes (Z-Mutation).

möglich. Rauchen ist sicherlich der wesentliche Manifestationsfaktor für die Lungenbeteiligung beim AATM. Wahrscheinlich erkranken viele Betroffene nicht klinisch manifest. Studien, die von Neugeborenen-Screening ausgehend über mehrere Jahrzehnte Betroffene mit Pi ZZ beobachtet hatten, zeigten, dass bis zum Alter von 40 Jahren nur ein kleiner Prozentsatz der Personen an Lebererkrankungen leidet und dass die Lunge bis zu diesem Zeitpunkt nicht beeinträchtigt ist [1517]. Auch existieren kaum Daten, die eine konkrete Prognoseabschätzung erlauben.

\section{Diagnostik}

Die Diagnose eines AATM erfolgt meist erst, nachdem Beschwerden bereits über mehrere Jahre bestanden haben. Hauptursache ist wohl die Seltenheit der Erkrankung. Es existieren verschiedene Empfehlungen, wann auf das Vorliegen eines AATM getestet werden soll. Nach einer WHO-Publikation sollen alle Patienten mit einer COPD untersucht werden [18], eine Leitlinie der ERS/ATS definiert weitere Zustände, z.B. Patienten mit neonatalem hepatischem Syndrom, Erwachsene mit chronischer Lebererkrankung und Familienmitglieder von Patienten mit AAT-Mangel [7]. Zunächst sollte die Bestimmung der AAT-Serumkonzentration erfolgen, bei Vorliegen erniedrigter Spiegel oder hochgradigem klinischem Verdacht sollte der Geno- und Phänotyp bestimmt werden. - Abb. 2 a fasst den Ablauf der Diagnostik zusammen.

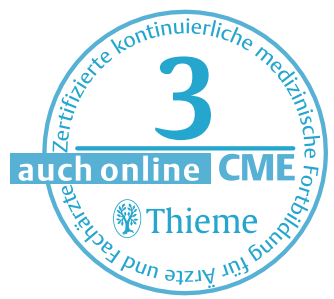




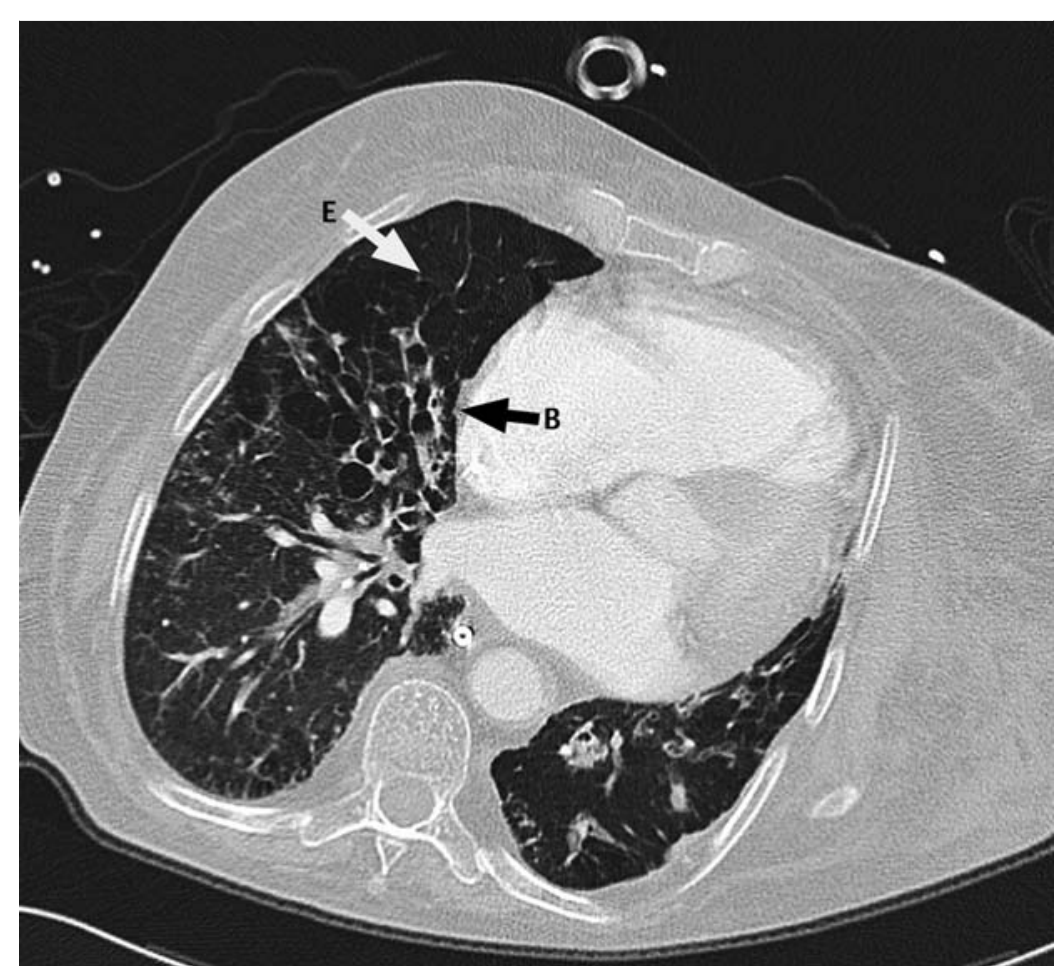

Abb. 3 Computertomographie der Lunge eines Patienten mit schwerer COPD. Es zeigen sich ein großblasiges Emphysem (E) sowie die Ausbildung von Bronchiektasen (B).

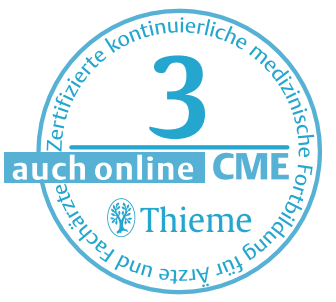

Zunächst sollte die Bestimmung der AAT-Serumkonzentration erfolgen, bei Vorliegen erniedrigter Spiegel oder hochgradigem klinischem Verdacht

Die Messung der Serum-Konzentration erlaubt eine gute Abgrenzung von schweren Mangelzuständen (z.B. Pi Z). Problematisch ist, dass das AAT ein Akutphaseprotein ist und bei generalisierten Entzündungsreaktionen auch bei Patienten mit einem mittelschweren Mangel (z.B. Pi $\mathrm{MZ}$ ) in normalen Konzentrationen vorliegt. Zum Ausschluss einer Entzündung kann der CRP-Spiegel (C-reaktives Protein) mitbestimmt werden. Unserer Erfahrung nach hat die Bestimmung der Serumkonzentration als initiales Testverfahren eine hohe Spezifität und Sensitivität zur Detektion eines schweren AATM [13]. Das Fehlen der alpha-1-Zacke in der Serumproteinelektrophorese ( $\bullet$ Abb. 2 b) ist zwar ein wichtiger Hinweis auf einen AATM, diese Methode wird jedoch nicht zur Diagnostik empfohlen.

Falls sich der Verdacht auf das Vorliegen eines AATM ergeben hat, muss der genaue Typ festgestellt werden, weil nur so eine Aussage über die Wahrscheinlichkeit einer Lebererkrankung und/ oder einer Gefährdung der Lunge getroffen werden kann. Hier stehen wiederum verschiedene Methoden zur Verfügung. Der sogenannte Phänotyp wird mittels isoelektrischer Fokusierung bestimmt ( $\bullet$ Abb. 2c). Dabei werden Serumproteine nach ihrem isoelektrischen Punkt aufgetrennt, das ist der pH, bei dem die Ladung des Moleküls sollten der Geno- und Phänotyp bestimmt werden. null ist. Diese Methode ist die klassische Methode zur Diagnose des AATM, ist jedoch arbeitsintensiv und bedarf einiger Erfahrung bei der Analyse. Der Genotyp kann über Allel-spezifische Polymerasekettenreaktionen (PCR) analysiert werden. Hierbei werden kommerzielle Verfahren angeboten, mit Hilfe derer die Existenz einer S- oder Z-Mutation nachgewiesen werden kann. Beim Vorliegen einer seltenen Mutation ist es oft notwendig, eine Gensequenzierung durchzuführen, bei der die Sequenz des SERPINA1 Gens untersucht wird ( $\bullet$ Abb. 2d). Letztendlich beinhaltet die Labordiagnose eines AATM die Kombination mehrerer Verfahren. Neben der Bestimmung der Serumkonzentration ist zur Diagnose die Durchführung einer Genotypisierung und ggf. einer Phänotypisierung notwendig.

Zur Diagnose und Stadieneinteilung der COPD sei zusätzlich auf die aktuelle DGP-Leitlinie verwiesen [19]. Kurz zusammengefasst gehören hierzu die Anamnese charakteristischer Symptome (chronischer Husten und Auswurf) sowie das Vorliegen bestimmter Risikofaktoren (Zigarettenrauchen) und der lungenfunktionsanalytische Nachweis einer nicht komplett reversiblen Atemwegsobstruktion. Des Weiteren sollten eine Ganzkörperplethysmographie und die Bestimmung der CO-Diffusionskapazität erfolgen. Bei ausgeprägter Belastungsdyspnoe sollte eine 6-Minuten-Gehstrecke sowie eine BGA in Ruhe und unter Belastung durchgeführt werden. Zur Differenzierung erfolgt zunächst eine Lungenfunktion mit Blutgasanalyse und ein Röntgen-Thorax in 2 Ebenen. Ein gegebenenfalls indiziertes CT-Thorax gibt Auskunft über das Vorliegen von Bullae und Bronchiektasen ( $\bullet$ Abb. 3).

Ein transthorakales Echokardiogramm erlaubt eine Aussage über eine Rechtsherzbelastung. Bezüglich der Lebererkrankung sollten bei unauffälligem Verlauf regelmäßige Bestimmungen der Transaminasen und eine Sonographie erfolgen. Bei Verdacht auf das Vorliegen einer Problematik in diesem Bereich ist eine Mitbetreuung durch einen hier spezialisierten Arzt anzustreben.

\section{Therapie der Lungenmanifestation $\nabla$}

Die Basistherapie der Lungenerkrankung beim AATM unterscheidet sich nicht vom Vorgehen bei einer AATM-unabhängigen COPD, hier sei auf die aktuelle Leitlinie verwiesen [19]. Kurz zusammengefasst besteht die Behandlung des Patienten aus einem multimodalen Konzept unter Einschluss von medikamentösen und nicht medikamentösen Verfahren. Das Erreichen einer Rauchabstinenz ist ein wichtiger Schritt [20]. Stadienabhängig kommen Bronchospasmolytika (BetaAgonisten, Anticholinergika) sowie in höheren Stadien inhalative Kortikosteroide zum Einsatz. Kommt es zu Exazerbationen, werden auch orale Kortikosteroide zeitlich begrenzt sowie bei Infekt- 
exazerbationen Antibiotika verwendet. Impfungen gegen Influenza und Pneumokokken sollten bei allen Patienten mit einer COPD erfolgen. Bei Hypoxämie kann eine Langzeitsauerstofftherapie erforderlich sein [21]. Die chirurgische Lungenvolumenreduktion ist aufgrund schlechter Langzeitergebnisse bei dieser Indikation verlassen worden [22]. Als ultima ratio besteht die Möglichkeit einer Lungentransplantation.

Eine spezifische Therapie des AATM ist die intravenöse Substitution des Alpha-1-Antitrypsins. Dies geschieht über wöchentliche Infusionen des Proteins, das von Plasmaspendern gewonnen wird. In den 80er-Jahren wurden Technologien entwickelt, um AAT aus Plasma zu isolieren. Die intravenöse Substitution führt zu einem Anstieg des Serumspiegels und zu messbaren Spiegeln in der bronchoalveolären Lavage (Review in [23]). In Registerkohorten wurden Mortalität und Abfall der Lungenfunktion bei substituierten und unsubstituierten Personen untersucht, wobei ein reduzierter FEV1-Abfall und eine verminderte Mortalität bei substituierten Patienten mit mäßiggradiger FEV1-Einschränkung gefunden wurden $[24,25]$. Ähnliche Befunde zeigten sich auch in einer Studie, in der der Lungenfunktionsverlauf vor und nach Beginn der Substitutionstherapie untersucht wurde [26]. Auch eine Vergleichsstudie mit dänischen (unsubstituierten) und deutschen (substituierten) Patienten bestätigte diese Befunde [27]. In den letzten Jahren wurde CT-basierte Densitometrie als Verlaufsparameter bei COPD insbesondere bei AATM-Patienten eingesetzt. In zwei Studien konnte gezeigt werden, dass dieses Messverfahren sehr sensitiv ist und dass die AAT-Substitution zu einer Verlangsamung des Verlustes an Lungendichte führt (statistisch signifikant, je nach Wahl des Auswerteverfahrens) $[28,29]$. Insbesondere zeigen diese Studien auch, dass neben klassischen Verlaufsparametern wie der FEV1 auch andere Messparameter in Studien die Effektivität einer COPD-Therapie messen können.

Angesichts der geschilderten Datenlage ist eine definitive Beurteilung des Therapieeffektes noch nicht möglich. Ausgehend von den bisherigen Daten ist die Indikation zur parenteralen Substitutionstherapie nach der aktuellen ATS/ERS Leitlinie unter folgenden Voraussetzungen gegeben:

- Schwerer AATM (Pi Z, Pi SZ, Nulltypen; Spiegel $<11 \mu \mathrm{M})$ und

- Mittelgradige Einschränkung der Lungenfunktion (FEV1 30-65\% Soll) oder rascher Abfall der FEV1 (> $120 \mathrm{ml} / \mathrm{Jahr}$ ) [7].

Außerhalb dieses Lungenfunktionsbereiches rechtfertigt die Datenlage nicht die Substitutionstherapie. Patienten, deren FEV1 während der Therapie unter $30 \%$ Soll absinkt, können gemäß der Leitlinie weitersubstituiert werden. Basierend auf der Pathophysiologie hat diese Art der Therapie keinen Einfluss auf die Lebererkrankung. Weitere Therapiemöglichkeiten sind in der Entwick-
Tab. 2 Ressourcen für die Versorgung von AAT-Betroffenen.

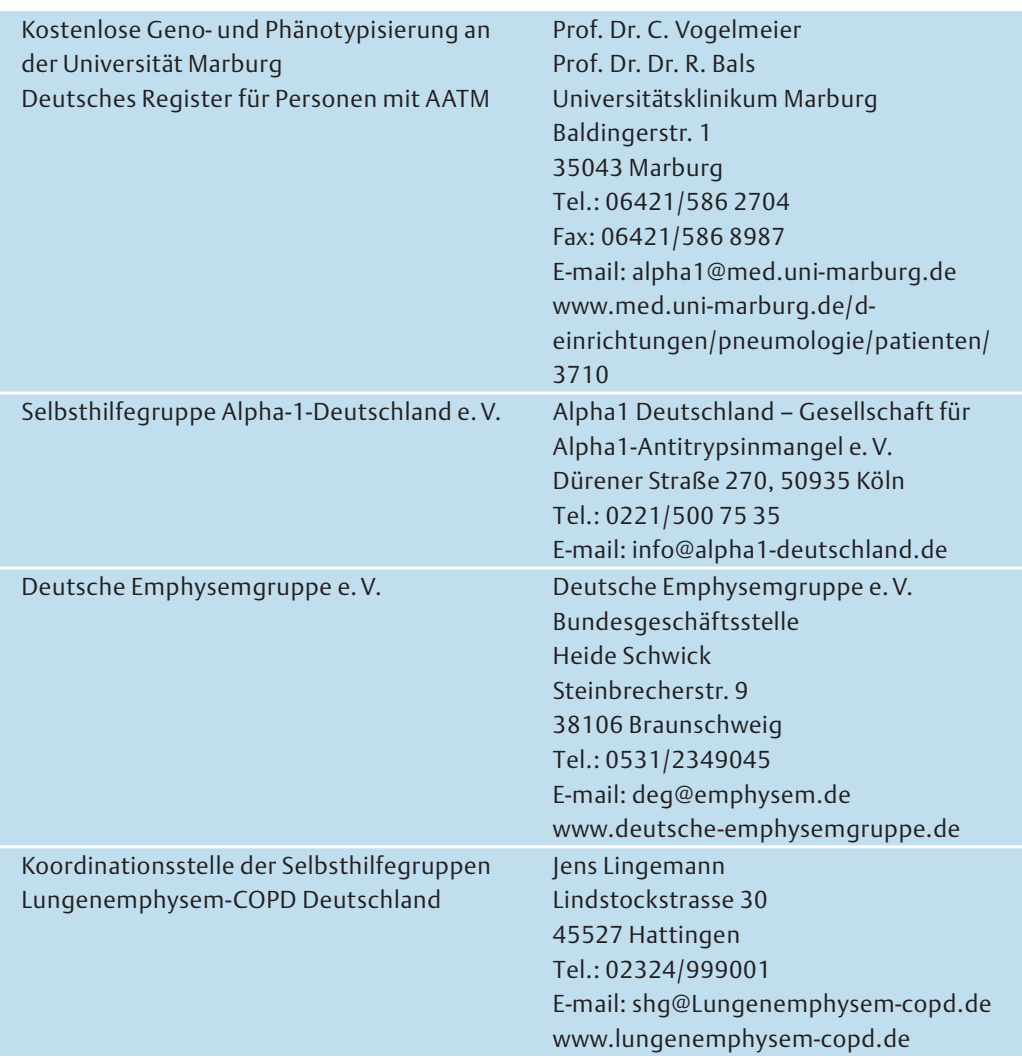

lung: Einige Pharmaunternehmen werden in der näheren Zukunft inhalierbares AAT in klinischen Studien testen. Synthetische und rekombinante Inhibitoren der Elastase sind ebenso in der Erprobung. Weitere Verfahren wie Gentherapie oder stammzellenbasierte Verfahren sind noch im präklinischen Stadium.

Für die Versorgung von AATM-Betroffenen ist auch der Aufbau von Registern und Forschungsnetzwerken wichtig, da nur so die Aktivitäten und Ressourcen zur Erforschung und Behandlung einer seltenen Erkrankung gezielt eingesetzt werden können. So existieren in Deutschland und auch im europäischen und US-amerikanischen Ausland Patientenregister $[11,30]$. Auch die Anbindung an Selbsthilfegruppen ist für Patienten ein wichtiger Schritt, um schnell mit anderen Betroffenen in Kontakt zu kommen ( $\bullet$ Tab. 2).

\section{Anhang}

In der Zusammenstellung in $\bullet$ Tab. 3 finden Sie die wichtigsten Informationen zur Diagnostik des Alpha-1-Antitrypsin-Mangels mit dem Fokus auf der Lungenmanifestation.

In der Zusammenstellung in $\bullet$ Tab. 4 finden Sie die wichtigsten Informationen zur Therapie der Lungenmanifestation des Alpha-1-Antitrypsinmangels. 
Tab. 3 Alpha-1-Antitrypsin Mangel (AATM). Diagnostik.

$\begin{array}{ll}\text { Biologie } & \text { Beim AATM kommt es durch Mutationen im SERPINA1 Gen zu } \\ & \text { einer verminderten und fehlerhaften Produktion des AAT } \\ & \text { Proteins. Dieses fehlgefaltete Eiweiß kann in Hepatozyten } \\ & \text { akkumulieren und so zu einer Leberzirrhose führen. Der niedrige } \\ & \text { Blutspiegel ist auch mit niedrigen Gewebespiegel in der Lunge } \\ \text { Wassoziert, was zu einem Lungenemphysem führen kann. }\end{array}$

Tab. 4 Alpha-1-Antitrypsin Mangel (AATM). Therapie.

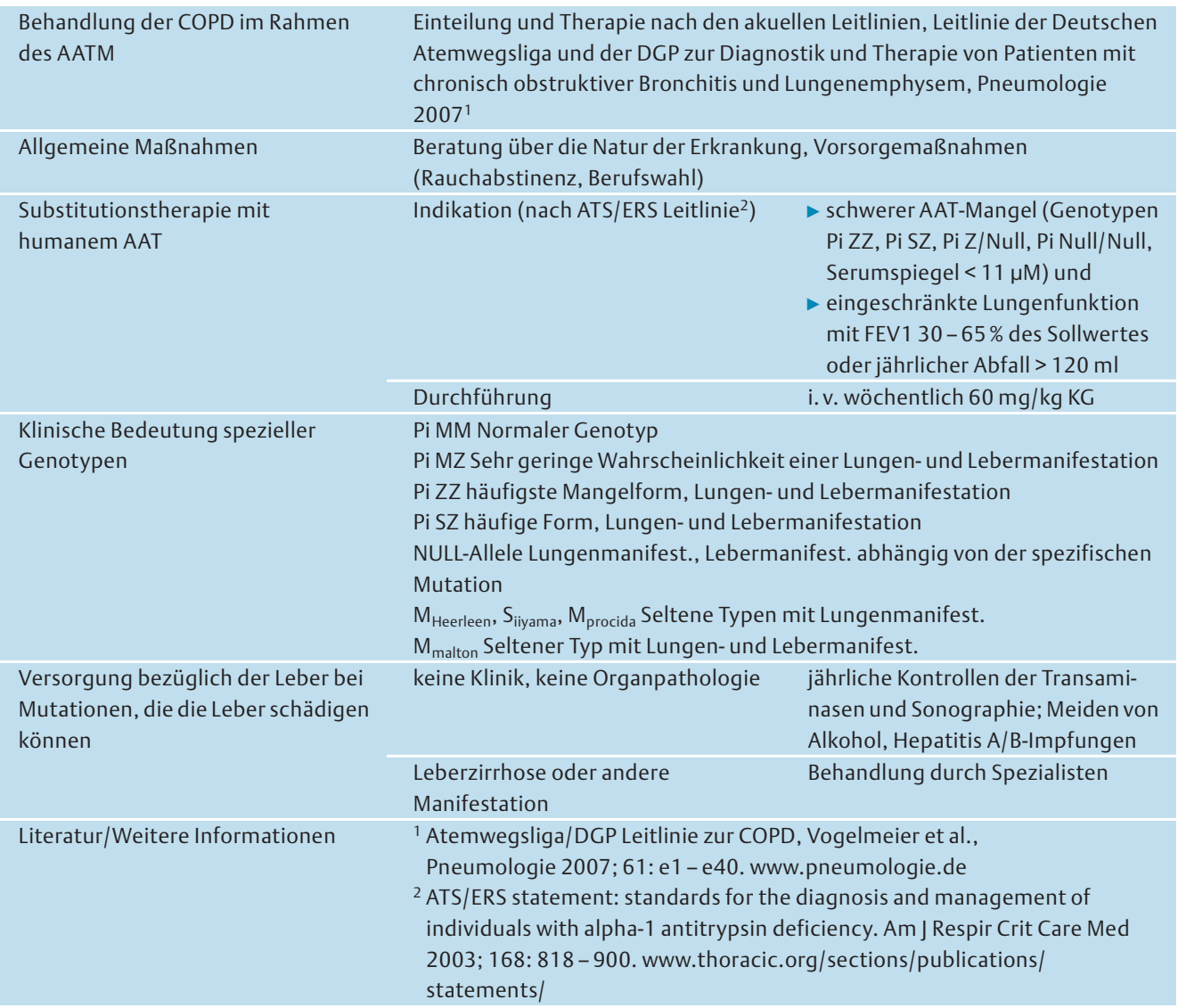




\section{Interessenkonflikt}

Wissenschaftliche Projekte des Klinikums werden seit 2003 von der Firma Bayer Healthcare/Talecris Biotherapeutics gefördert. KR, CV und RB erhielten Vortragshonorare und Reisesponsoring durch diese Firmen. Die Präsentation des Themas ist unabhängig und die Darstellung des Inhalts produktneutral.

\section{Literatur}

1 Campbell E], Campbell MA, Boukedes SS et al. Quantum proteolysis by neutrophils: implications for pulmonary emphysema in alpha 1-antitrypsin deficiency. J Clin Invest 1999; 104: $337-344$

2 Fairbanks KD, Tavill AS. Liver disease in alpha 1-antitrypsin deficiency: a review. Am J Gastroenterol 2008; 103: $2136-2141$

3 Stoller JK, Aboussouan LS. Alpha1-antitrypsin deficiency. Lancet 2005; 365: 2225-2236

4 Mahadeva R, Lomas DA. Genetics and respiratory disease. 2. Alpha 1-antitrypsin deficiency, cirrhosis and emphysema. Thorax 1998; 53: 501-505

5 Lomas DA, Parfrey H. Alpha1-antitrypsin deficiency. 4: Molecular pathophysiology. Thorax 2004; 59: 529-535

6 Crystal RG. Alpha 1-antitrypsin deficiency, emphysema, and liver disease. Genetic basis and strategies for therapy. J Clin Invest 1990; 85: 1343-1352

7 American Thoracic Society/European Respiratory Society Statement. Standards for the Diagnosis and Management of Individuals with Alpha-1 Antitrypsin Deficiency. Am J Respir Crit Care Med 2003; 168: 818-900

8 DeMeo DL, Silverman EK. Alpha1-antitrypsin deficiency. 2: genetic aspects of alpha(1)-antitrypsin deficiency: phenotypes and genetic modifiers of emphysema risk. Thorax 2004; 59: 259-264

9 Mahadeva R, Atkinson C, Li Z et al. Polymers of Z alpha1antitrypsin co-localize with neutrophils in emphysematous alveoli and are chemotactic in vivo. Am J Pathol 2005; 166: 377-386

10 Blanco I, de Serres F], Fernandez-Bustillo E et al. Estimated numbers and prevalence of $\mathrm{PI}^{*} \mathrm{~S}$ and $\mathrm{PI}^{*} \mathrm{Z}$ alleles of alpha1-antitrypsin deficiency in European countries. ERJ 2006; 27: 77-84

11 Stockley RA, Luisetti M, Miravitlles $M$ et al. Ongoing research in Europe: Alpha One International Registry (AIR) objectives and development. ERI 2007; 29: 582 - 586

12 McElvaney NG, Stoller JK, Buist AS et al. Baseline characteristics of enrollees in the National Heart, Lung and Blood Institute Registry of alpha 1-antitrypsin deficiency. Alpha 1-Antitrypsin Deficiency Registry Study Group. Chest 1997; 111: 394-403

13 Bals $R$, Koczulla $R$, Kotke $V$ et al. Identification of individuals with alpha-1-antitrypsin deficiency by a targeted screening program. Respir Med 2007; 101: 1217-1218

14 Campos MA, Wanner A, Zhang G, Sandhaus RA. Trends in the diagnosis of symptomatic patients with alpha1-antitrypsin deficiency between 1968 and 2003. Chest 2005; 128: $1179-1186$

15 Sveger $T$. Liver disease in alpha1-antitrypsin deficiency detected by screening of 200000 infants. N Engl J Med 1976; 294: 1316-1321

16 Piitulainen E, Sveger T. Effect of environmental and clinical factors on lung function and respiratory symptoms in adolescents with alpha1-antitrypsin deficiency. Acta Paediatr 1998; 87(11): 1120-1124

17 Bernspang E, Sveger T, Piitulainen E. Respiratory symptoms and lung function in 30-year-old individuals with alpha-1-antitrypsin deficiency. Respir Med 2007; 101: $1971-1976$
18 Anonymous. Alpha 1-antitrypsin deficiency: memorandum from a WHO meeting. Bull World Health Organ 1997; 75: $397-415$

19 Vogelmeier C, Buhl R, Criée CP et al. Leitlinie der Deutschen Atemwegsliga und der Deutschen Gesellschaft für Pneumologie und Beatmungsmedizin zur Diagnostik und Therapie von Patienten mit chronisch obstruktiver Bronchitis und Lungenemphysemen (COPD). Pneumologie 2007; 61: e1 - e440

20 Andreas S, Batra A, Behr J et al. Tabakentwöhnung bei COPD. S3 Leitlinie herausgegeben von der Deutschen Gesellschaft für Pneumologie und Beatmungsmedizin. Pneumologie 2008; 62: 255-272

21 Magnussen H, Goeckenjan G, Kohler D et al. Leitlinien zur Langzeit-Sauerstofftherapie. Pneumologie 2001; 55: $454-464$

22 Tutic M, Bloch KE, Lardinois D et al. Long-term results after lung volume reduction surgery in patients with alpha1antitrypsin deficiency. J Thorac Cardiovasc Surg 2004; 128: $408-413$

23 Sandhaus RA. alpha1-Antitrypsin deficiency. 6: new and emerging treatments for alpha1-antitrypsin deficiency. Thorax 2004; 59: 904 - 909

24 Wencker M, Banik N, Buhl R et al. Long-term treatment of alpha1-antitrypsin deficiency-related pulmonary emphysema with human alpha1-antitrypsin. Wissenschaftliche Arbeitsgemeinschaft zur Therapie von Lungenerkrankungen (WATL)-alpha1-AT-study group. Eur Respir J 1998; 11: 428-433

25 Alpha-1-Antitrypsin Deficiency Registry Study Group. Survival and FEV1 decline in individuals with severe deficiency of alpha1-antitrypsin. The Alpha-1-Antitrypsin Deficiency Registry Study Group. Am J Respir Crit Care Med 1998; 158: 49-59

26 Wencker M, Fuhrmann B, Banik $N$ et al. Longitudinal follow-up of patients with alpha(1)-protease inhibitor deficiency before and during therapy with IV alpha(1)-protease inhibitor. Chest 2001; 119: 737-744

27 Seersholm N, Wencker M, Banik N et al. Does alpha1-antitrypsin augmentation therapy slow the annual decline in FEV1 in patients with severe hereditary alpha1-antitrypsin deficiency? Wissenschaftliche Arbeitsgemeinschaft zur Therapie von Lungenerkrankungen (WATL) alpha1AT study group. Eur Respir J 1997; 10: 2260 - 2263

28 Dirksen A, Dijkman JH, Madsen F et al. A randomized clinical trial of alpha(1)-antitrypsin augmentation therapy. Am J Respir Crit Care Med 1999; 160: 1468 - 1472

29 Dirksen A, Piitulainen E, Parr DG et al. Exploring the role of CT densitometry: a randomised study of augmentation therapy in alpha-1 antitrypsin deficiency. Eur Respir J 2009; Feb. 5 [Epub]

30 Koczulla R, Bittkowski N, Andress J et al. Das Deutsche Register für Personen mit Alpha-1-Antitrypsin-Mangel eine Ressource für die Versorgungsforschung. Pneumologie 2008; 62: 655-658 


\section{CME-Fragen Alpha-1-Antitrypsin-Mangel: Diagnose und Therapie der pulmonalen Erkrankung}

\section{Welche Aussage zu AAT ist richtig?}
A AAT ist ein Serumproteinaseinhibitor, welcher die körpereigenen Proteine vor der Verdauung schützt.
B AAT wird vor allem in der Lunge produziert.
C AAT wird vor allem in den Nieren produziert.
D AAT wird über die Lymphflüssigkeit im Körper verteilt.
E AAT ist kein Akute-Phase-Protein.

2 Welche Aussage zum AAT-Mangel ist richtig?

A Der AAT-Mangel ist eine Erkrankung, die nur durch äußere Umwelteinflüsse entsteht.

B Der AAT-Mangel hat als Konsequenz nur das Lungenparenchym.

C Der AAT-Mangel kann zur Entwicklung einer Leberzirrhose führen.

D Der AAT-Mangel führt zu einem Mangel an Oxidantien.

E Der AAT-Mangel betrifft v.a. die asiatischen Rassen.

3 Die AAT-Mutationen
A bewirken eine inadäquat erhöhte Produktion an AAT.
B führen zu einem Ungleichgewicht zwischen Anti- proteasen und Proteinasen.
C haben immer eine komplett fehlende AAT-Wirkung als Folge.
D umfassen eine Vielzahl von Mutationen, die klinisch gleich relevant sind.
E können nicht voneinander unterschieden werden.

4 Was trifft zur Epidemiologie des AATM zu?

A In Deutschland sind ca. 80000-120000 Menschen an schwerem AATM erkrankt.

B Die Prävalenz des schweren Mangels in den mitteleuropäischen Ländern beträgt etwa 1-2\%.

C In Asien stellt der Alpha-1-Antitrypsinmangel die häufigste autosomal vererbbare Krankheit dar, die zum Tode führen kann.

D Es sind ungefähr 2500 Patienten mit einem AATM bekannt.

E Bei Patienten mit einer COPD ist der AATM nicht häufiger als in der Allgemeinbevölkerung.
5 Welche Aussage bezüglich der Symptomatik der Lungenmanifestation trifft zu?

A Die Beschwerden einer AATM-bedingten COPD unterscheiden sich deutlich von denen einer konventionellen COPD.

B Die typischen ersten Beschwerden sind Husten und Belastungsdyspnoe.

D Die Diagnose bei Auftreten der Symptome wird sehr rasch gestellt.

C Bei AATM ist je nach Genotyp eine genaue Aussage über den Verlauf der Erkrankung möglich.

E Die Beschwerden von Seiten der Lungenerkrankung treten typischerweise erst nach dem 70. Lebensjahr auf.

6 Welche Aussage zum diagnostischen Algorithmus trifft zu?

A Nach einer WHO-Empfehlung sollten alle Patienten mit einer COPD untersucht werden.

B Zunächst sollte der Geno- und Phänotyp bestimmt werden, danach die Serumkonzentration.

C Die Messung der Serumkonzentration erlaubt keine gute Abgrenzung von schweren Mangelzuständen.

D Die Bestimmung der Serumkonzentration als initiales Testverfahren ist nicht aussagekräftig.

E Die Durchführung einer Serumproteinelektrophorese ist der initiale Diagnoseschritt.

7 Was trifft zur Diagnostik des AATM zu?

A Die AAT-Konzentration im Serum wird kaum durch Entzündungen beeinflusst.

B Beim Vorliegen eines AATM soll der genaue Typ festgestellt werden, weil nur so eine Aussage über die Wahrscheinlichkeit einer Lebererkrankung und einer Gefährdung der Lunge getroffen werden kann.

C Der sogenannte Phänotyp wird mittels PCR bestimmt.

D Eine spezifische Diagnostik der COPD ist bei vorliegendem Genotyp nicht mehr notwendig.

E Bei Befall der Leber muss ein Leberbiopsie durchgeführt werden. 
8 Welche Aussage bezüglich der Therapie der Lungenmanifestation trifft zu?

A Die Grundtherapie der Lungenerkrankung beim AATM unterscheidet sich wesentlich vom Vorgehen bei einer AATM-unabhängigen COPD.

B Das Erreichen einer Rauchabstinenz hat aufgrund eines weiterhin bestehenden AATM keinen Einfluss auf die Erkrankung.

c Eine Exazerbation ist immer eine Indikation für eine Substitutionstherapie.

D Eine prophylaktische Impfung gegen Pneumokokken und jährlich gegen Grippe ist bei Personen mit AATM wirkungslos.

E Stadienabhängig kommen Bronchospasmolytika (Beta-Agonisten, Anticholinergika) sowie in höheren Stadien inhalative Kortikosteroide zum Einsatz.

9 Welche Aussage bezüglich der Substitutionstherapie ist richtig?

A Eine spezifische Therapie des AATM ist die orale Substitution des AAT.

B Die Substitution des AAT wird monatlich durchgeführt.

C Jede Person mit einem sehr niedrigen AATM-Serumspiegel soll eine Substitution erhalten.

D Gemäß der ATS/ERS Leitlinie soll eine Substitution bei einer FEV1 von 30-65 \% oder einem schnellen Abfall erfolgen.

E Basierend auf der Pathophysiologie hat die AAT-Substitution einen Einfluss auf die Leberund Lungenerkrankung.
10

Welche Aussage ist richtig?

A Für die Versorgung von AATM-Betroffenen gibt es noch keine nationalen und internationalen Register.

B Die Kontaktaufnahme mit Selbsthilfegruppen ist für Patienten ein wichtiger Schritt, um schnell mit anderen Betroffenen in Kontakt zu kommen.

C Die Kosten einer AAT-Substitution sind gering.

D Der Alpha-1-Antitrypsinmangel (AATM) ist eine X-chromosomal-dominant vererbbare Erkrankung.

E Die genetisch bedingte Konformationsänderung des AAT kann zu einer Akkumulation in der Lunge und zu einem Mangel im übrigen Organismus führen.

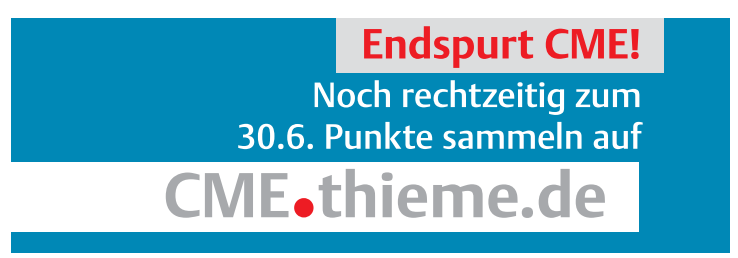


A Angaben zur Person

\section{B Lernerfolgskontrolle}

Bitte nur eine Antwort pro Frage ankreuzen

ankreuzen
Name, Vorname, Titel:

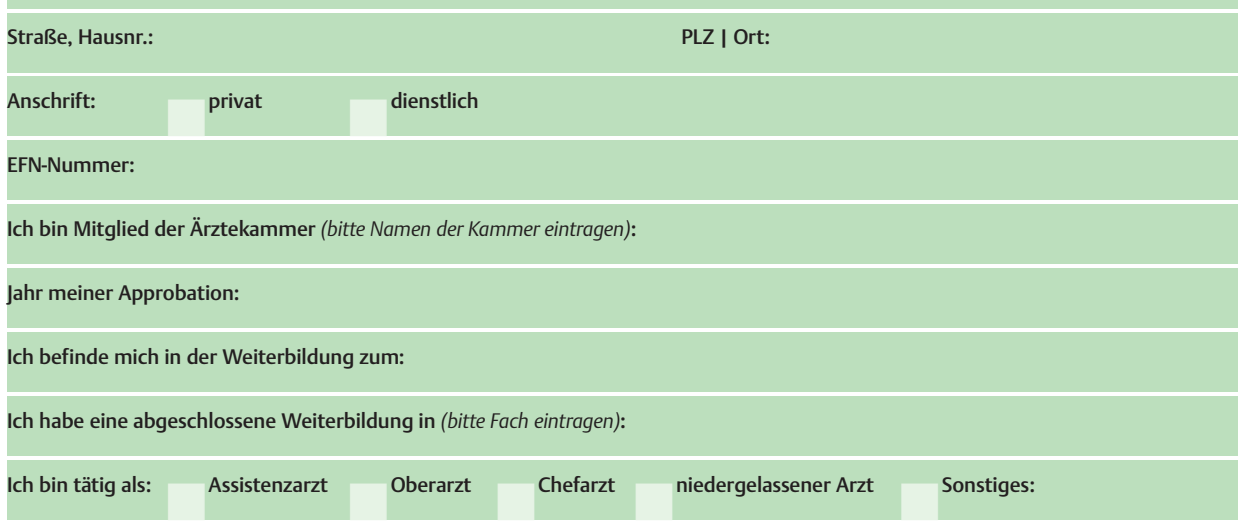

\section{1}

\section{2}

3

4

5
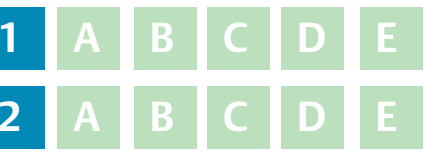

3 A B C D E

4 A B C D E

5 A B C D E
6 A $B$ C D E

7 A

8 A

9 A

10 A B C D E

\section{Erklärung}

Ich versichere, dass ich die Beantwortung der Fragen selbst und ohne Hilfe durchgeführt habe

Ort | Datum: $\quad$ Unterschrift:

Deld für CME-Wertmarke

Bitte unbedingt nächste

Seite ausfüllen!
E Zertifizierungsfeld

Bitte in dieses Feld die CME-Wertmarke kleben oder Ihre Abonnement-Nummer eintragen: (siehe Adressaufkleber)

Zertifizierungsfeld (wird durch den Verlag ausgefüllt)

\section{Ihr Ergebnis}

Sie haben
Sie haben von 10 Fragen richtig beantwortet.

bestanden und 3 CME-Punkte erworben.

nicht bestanden

Stuttgart, den

Datum

Stempel/Unterschrift 


\section{F Fragen zur Zertifizierung}

Eine Antwort pro Frage. Bitte unbedingt ausfüllen bzw. ankreuzen, da die Evaluation sonst unvollständig ist!

\section{Didaktisch-methodische Evaluation}

1 Das Fortbildungsthema kommt in meiner ärztlichen Tätigkeit häufig vor regelmäßig vor selten vor gar nicht vor

2 Zum Fortbildungsthema habe ich eine feste Gesamtstrategie noch offene Einzelprobleme: keine Strategie

3 Hinsichtlich des Fortbildungsthemas

fühle ich mich nach dem Studium des Beitrags in meiner Strategie bestätigt habe ich meine Strategie verändert: habe ich erstmals eine einheitliche Strategie erarbeitet habe ich keine einheitliche Strategie erarbeiten können

4 Wurden aus der Sicht Ihrer täglichen Praxis heraus wichtige Aspekte des Themas nicht erwähnt? zu knapp behandelt? ja, welche nein überbewertet? ja, welche ja, welche nein nein

5 Verständlichkeit des Beitrags

Der Beitrag ist nur für Spezialisten verständlich

Der Beitrag ist auch für Nicht-Spezialisten verständlich

6 Beantwortung der Fragen

Die Fragen lassen sich aus dem Studium des Beitrages allein beantworten

Die Fragen lassen sich nur unter Zuhilfenahme zusätzlicher Literatur beantworten

7 Die Aussagen des Beitrages benötigen eine ausführlichere Darstellung zusätzlicher Daten von Befunden bildgebender Verfahren die Darstellung ist ausreichend

8 Wie viel Zeit haben Sie für das Lesen des Beitrages und die Bearbeitung des Quiz benötigt?
$>$ Einsendeschluss 15.6. 2010
Bitte senden Sie den vollständigen Antwortbogen zusammen mit einem an Sie selbst adressierten und ausreichend frankierten Rückumschlag an den

Georg Thieme Verlag KG, Stichwort „CME“, Postfach 301120,70451 Stuttgart 